\title{
Group theoretical interpretation of the modified gravity in de Sitter space
}

\author{
M. Dehghani \\ Department of Physics, Ilam University, \\ Ilam, Iran \\ E-mail: m.dehghani@ilam.ac.ir
}

ABSTRACT: A framework has been presented for theoretical interpretation of various modified gravitational models which is based on the group theoretical approach and unitary irreducible representations (UIR's) of de Sitter (dS) group. In order to illustrate the application of the proposed method, a model of modified gravity has been investigated. The background field method has been utilized and the linearized modified gravitational field equation has been obtained in the 4-dimensional dS space-time as the background. The field equation has been written as the eigne-value equation of the Casimir operators of dS space using the flat 5-dimensional ambient space notations. The Minkowskian correspondence of the theory has been obtained by taking the zero curvature limit. It has been shown that under some simple conditions, the linearized modified field equation transforms according to two of the UIR's of dS group labeled by $\Pi_{2,1}^{ \pm}$and $\Pi_{2,2}^{ \pm}$in the discrete series. It means that the proposed modified gravitational theory can be a suitable one to describe the quantum gravitational effects in its linear approximation on dS space. The field equation has been solved and the solution has been written as the multiplication of a symmetric rank-2 polarization tensor and a massless scalar field using the ambient space notations. Also the two-point function has been calculated in the ambient space formalism. It is dS invariant and free of any theoretical problems.

Keywords: Classical Theories of Gravity, Models of Quantum Gravity

ArXIV EPRINT: 1601.03946 


\section{Contents}

1 Introduction 1

2 The field equation $\quad 2$

2.1 Linear field equation in dS space 3

2.2 dS group and Casimir operators in the field equation 5

3 Solution to the conformal field equation $\quad 9$

4 The conformal two-point function $\quad 12$

5 Conclusion 13

$\begin{array}{ll}\text { A Some useful mathematical relations } & 14\end{array}$

$\begin{array}{ll}\text { B Details of derivation of eq. (2.2) } & \mathbf{1 5}\end{array}$

C Details of derivation of eq. (2.7) 16

$\begin{array}{ll}\text { D Details of derivation of eq. (2.9) } & \mathbf{1 7}\end{array}$

$\begin{array}{ll}\text { E Details of derivation of eq. (2.11) } & \mathbf{1 7}\end{array}$

$\begin{array}{ll}\text { F Details of derivation of eq. (3.3) } & 18\end{array}$

\section{Introduction}

It is well known that there are many good reasons to consider the Einstein general relativity as the best theory for the gravitational interaction, but according to the recent cosmological observations it seems that this theory may be incomplete. In addition to the well known problems of the Einstein general relativity in explaining the astrophysical phenomenology (i.e., the galactic rotation curves and small scale structure formation), recent cosmological data indicate an underlying cosmic acceleration of the universe which cannot be recast in the framework of the Einstein general relativity.

It is for these reasons and some other issues such as cosmic microwave background anisotropies [1, 2], large scale structure formation [3, 4], baryon oscillations [5] and weak lensing [6] that in recent years many authors are interested to generalize standard Einstein gravity. Among alternative proposed models the so-called extended theory of gravitation and, in particular, the gravity theories stem from nonlinear actions or higher-order theories of gravity have provided interesting results [7-19]. These models are based on gravitational 
actions which are non-linear in the Ricci curvature $\mathcal{R}$ and/ or contain terms involving combinations of derivatives of $\mathcal{R}$ [20-23].

Recent astronomical observations of supernova and cosmic microwave background [2427] indicate that the universe is accelerating and can be well approximated by a world with a positive cosmological constant. If the universe accelerates indefinitely, the standard cosmology leads to an asymptotic dS universe. In addition, dS space-time plays an important role in the inflationary scenario where an exponentially expanding approximately dS spacetime is employed to solve a number of problems in standard cosmology. Furthermore, the quantum field theory on dS space-time is also of considerable interest.

Furthermore, the gravitational field in the linear approximation behaves like a massless spin-2 particle which propagates on the background space-time. Following the Wigner's theorem, a linear gravitational field should transform according to the UIR's of the symmetric group of the background space-time. In this paper, dS space-time has been considered as the background. It has been shown that the proposed generalized Einstein's theory, in its linear approximation, can be associated with the UIR's of dS group.

The main goal of this work is to propose a theoretical framework for validity interpretation of the modified gravity theories, from group theoretical point of view, in dS space. The idea is that if a proposed model of modified gravity corresponds to the UIR,s of dS group it can be considered as a possible successful model.

The organization of this paper is based on the following order. In section-2, a generalized Einstein-Hilbert gravitational action has been introduced and corresponding linear generalized Einstein gravitational field equation has been obtained in terms of the intrinsic dS coordinates as the background. Details of derivations have been given in appendices. Next, the linearized field equation has been written in terms of the Casimir operators of dS group making use of the five-dimensional ambient space formalism. The physical sector of the theory has been obtained by imposing the divergenceless and traceless conditions and the possible relations between this field equation and the UIR's of dS group have been investigated. By imposing a simple condition the conformally invariant theory of gravity is reproduced. In section-3, we obtained the solution to the conformally invariant field equation, using the ambient space notations. The solution can be written as the multiplication of a symmetric generalized polarization rank-2 tensor and a massless minimally coupled scalar field in dS space. In section-4, we have calculated the conformally invariant twopoint function, in terms of the massless minimally coupled scalar two-point function, using the ambient space formalism. It is dS invariant, symmetric and satisfies the traceless and divergenceless conditions. The results are summarized and discussed in section-5. Some useful mathematical relations and details of derivations of equations have been given in the appendices.

\section{The field equation}

The terms containing fourth order derivatives of the metric may be constructed out by curvature invariants (other than the cosmological constant), that is

$$
\mathcal{R}, \quad \mathcal{R}^{2}, \quad \mathcal{R}_{a b} \mathcal{R}^{a b}, \quad \mathcal{R}_{a b c d} \mathcal{R}^{a b c d} .
$$


Therefore, the gravitational action for the modified field equation in the 4-dimensional $\mathrm{dS}$ space-time with the metric signature $(-,+,+,+)$ can be written in the following general form

$$
I=\frac{1}{16 \pi G} \int d^{4} x \sqrt{-g}\left[a_{0}(\mathcal{R}-2 \Lambda)+a_{1} \mathcal{R}^{2}+a_{2} \mathcal{R}^{a b} \mathcal{R}_{a b}+a_{3} \mathcal{R}^{a b c d} \mathcal{R}_{a b c d}\right],
$$

where $\Lambda=3 H^{2}$ is the positive cosmological constant. $\mathcal{R}_{a b c d}$ is the Riemann tensor, $\mathcal{R}_{a b}$ is the Ricci tensor and $\mathcal{R}=g^{a b} \mathcal{R}_{a b}$ is the Ricci scalar of the space-time under consideration. $a_{0}, a_{1}, a_{2}$ and $a_{3}$ are constant coefficients. The coefficients $a_{1}, a_{2}$ and $a_{3}$ are positive with the dimension of (Length) ${ }^{2}$.

Taking note the fact that the Gauss-Bonnet action

$$
\frac{1}{16 \pi G} \int d^{4} x \sqrt{-g}\left(\mathcal{R}^{2}-4 \mathcal{R}^{a b} \mathcal{R}_{a b}+\mathcal{R}^{a b c d} \mathcal{R}_{a b c d}\right)
$$

is a total divergence. Adding it to the action will not contribute to the field equations and enable us to simplify the action somewhat and rewrite it as

$$
I=\frac{1}{16 \pi G} \int d^{4} x \sqrt{-g}\left[a_{0}(\mathcal{R}-2 \Lambda)+a \mathcal{R}^{2}+b \mathcal{R}^{a b} \mathcal{R}_{a b}\right],
$$

with new coefficients. Therefore, including an $\mathcal{R}^{a b c d} \mathcal{R}_{a b c d}$ term is equivalent to altering the coefficients. The theory described by this action is referred to as fourth-order gravity, since it leads to fourth order equations. Numerous papers have been devoted to the study of fourth-order gravity.

Varying the action (2.1) with respect to the metric tensor $g_{a b}$ the modified gravitational field equation is obtained as (appendix B)

$$
a_{0} \mathcal{H}_{a b}^{(0)}+a \mathcal{H}_{a b}^{(1)}+b \mathcal{H}_{a b}^{(2)}=0
$$

where $\mathcal{H}_{a b}^{(0)}=G_{a b}+\Lambda g_{a b}$ and $G_{a b}=\mathcal{R}_{a b}-\frac{1}{2} \mathcal{R} g_{a b}$ is the Einstein tensor and

$$
\begin{aligned}
& \mathcal{H}_{a b}^{(1)}=2 \mathcal{R} \mathcal{R}_{a b}-2 \nabla_{a} \nabla_{b} \mathcal{R}-\frac{1}{2} g_{a b}\left(\mathcal{R}^{2}-4 \square \mathcal{R}\right), \\
& \mathcal{H}_{a b}^{(2)}=\square \mathcal{R}_{a b}-\nabla_{c} \nabla_{a} \mathcal{R}_{b}^{c}-\nabla_{c} \nabla_{b} \mathcal{R}_{a}^{c}+2 \mathcal{R}_{a}^{c} \mathcal{R}_{c b}-\frac{1}{2} g_{a b}\left(\mathcal{R}^{c d} \mathcal{R}_{c d}-2 \nabla_{c} \nabla_{d} \mathcal{R}^{c d}\right)
\end{aligned}
$$

Making use of the relations $\left[\nabla_{c}, \nabla_{a}\right] \mathcal{R}_{b}^{c}=\mathcal{R}_{d a} \mathcal{R}_{b}^{d}-\mathcal{R}_{b c a}^{d} \mathcal{R}_{d}^{c}, \nabla_{c} \mathcal{R}_{b}^{c}=1 / 2 \nabla_{b} \mathcal{R}, \nabla_{c} \nabla_{d} \mathcal{R}^{c d}=$ $1 / 2 \square \mathcal{R}$ and other symmetry properties of the Riemann tensor [19], it is easy to show that the field equation (2.2) is agree with eq. (2.3) of ref. [28] with $\gamma=0$.

\subsection{Linear field equation in dS space}

In order to obtain the linearized form of the field equation (2.2), one can use the background field method. That is $g_{a b}=g_{a b}^{(B G)}+h_{a b}$, in which $g_{a b}^{(B G)}$ is the background metric and $h_{a b}$ is its fluctuations. Indices are raised and lowered by the background metric. We suppose that $g_{a b}^{(B G)}=g_{a b}^{(d s)} \equiv \tilde{g}_{a b}$. So one can write

$$
g_{a b}=\tilde{g}_{a b}+h_{a b} \quad \text { and } \quad g^{a b}=\tilde{g}^{a b}-h^{a b} .
$$


The metric $\tilde{g}_{a b}$ is a solution to Einstein's field equation with the positive cosmological constant $\Lambda=3 H^{2}$ :

$$
\tilde{R}_{a b}-\frac{1}{2} \tilde{R} \tilde{g}_{a b}+3 H^{2} \tilde{g}_{a b}=0 .
$$

Using the approximations given in eq. (2.5), in eq. (2.3), we have (appendix C)

$$
\mathcal{H}_{a b}^{(0)}=\tilde{H}_{a b}^{(0)}+H_{a b}^{(0)}
$$

where $\tilde{H}_{a b}^{(0)}$ is the dS correspondent to $\mathcal{H}_{a b}^{(0)}$ and

$$
\begin{aligned}
H_{a b}^{(0)}= & \frac{1}{2}\left(\nabla_{a} \nabla^{c} h_{b c}+\nabla_{b} \nabla^{c} h_{a c}-\square h_{a b}-\nabla_{a} \nabla_{b} h^{\prime}+2 H^{2} h_{a b}\right) \\
& +\frac{1}{2} \tilde{g}_{a b}\left(\square h^{\prime}-\nabla_{c} \nabla_{d} h^{c d}+H^{2} h^{\prime}\right),
\end{aligned}
$$

in which $h^{\prime}=h_{a}^{a}$ is the trace of $h_{a b}$ with respect to the background metric and $\nabla^{b}$ is the background covariant derivative. It is easy to show that (appendix D)

$$
\mathcal{H}_{a b}^{(1)}=\tilde{H}_{a b}^{(1)}+H_{a b}^{(1)}
$$

where $\tilde{H}_{a b}^{(1)}$ is the correspondent to $\mathcal{H}_{a b}^{(1)}$ in dS space and

$$
\begin{aligned}
H_{a b}^{(1)}= & +12 H^{2}\left(\nabla_{a} \nabla^{c} h_{b c}+\nabla_{b} \nabla^{c} h_{a c}-\square h_{a b}\right)-2 \nabla_{a} \nabla_{b}\left(\nabla_{c} \nabla_{d} h^{c d}-\square h^{\prime}+3 H^{2} h^{\prime}\right) \\
& +24 H^{4} h_{a b}-2 \tilde{g}_{a b}\left(3 H^{2} \nabla_{c} \nabla_{d} h^{c d}+3 H^{4} h^{\prime}-\square \nabla_{c} \nabla_{d} h^{c d}+\square^{2} h^{\prime}\right) .
\end{aligned}
$$

It is easy to show that (appendix E)

$$
\mathcal{H}_{a b}^{(2)}=\tilde{H}_{a b}^{(2)}+H_{a b}^{(2)}
$$

where $\tilde{H}_{a b}^{(2)}$ is the correspondent to $\mathcal{H}_{a b}^{(2)}$ in dS space and

$$
\begin{aligned}
H_{a b}^{(2)}= & \frac{1}{2}\left[\square\left(\nabla_{a} \nabla_{c} h_{b}^{c}+\nabla_{b} \nabla_{c} h_{a}^{c}\right)-2 H^{2} \square h_{a b}-\square^{2} h_{a b}+\nabla_{a} \nabla_{b} \square h^{\prime}\right] \\
& +2 H^{2}\left(\nabla_{a} \nabla_{c} h_{b}^{c}+\nabla_{b} \nabla_{c} h_{a}^{c}\right)-\nabla_{a} \nabla_{b} \nabla_{c} \nabla_{d} h^{c d}-3 H^{2} \nabla_{a} \nabla_{b} h^{\prime}+4 H^{4} h_{a b} \\
& +\frac{1}{2} \tilde{g}_{a b}\left(2 H^{2} \nabla_{c} \nabla_{d} h^{c d}-2 H^{4} h^{\prime}+7 H^{2} \square h^{\prime}+\square \nabla_{c} \nabla_{d} h^{c d}-\square^{2} h^{\prime}\right) .
\end{aligned}
$$

Substituting eqs. (2.8), (2.10) and (2.12) in eq. (2.2), we have

$$
a_{0} H_{a b}^{(0)}+a H_{a b}^{(1)}+b H_{a b}^{(2)}=0 .
$$

eq. (2.13) is the linearized modified gravitational field equation in dS background, which has been written in terms of the intrinsic coordinates $X_{a}$ of the 4-dimensional dS space-time. 
The linear field equation (2.13) can be written in the following explicit form

$$
\begin{aligned}
-\frac{b}{2} \square^{2} h_{a b}-\left(\frac{a_{0}}{2}+12 a H^{2}+b H^{2}\right) \square h_{a b}+H^{2}\left(a_{0}+24 a H^{2}+4 b H^{2}\right) h_{a b} \\
+\left(2 a+\frac{b}{2}\right) \nabla_{a} \nabla_{b} \square h^{\prime}-\left(\frac{a_{0}}{2}+6 a H^{2}+3 b H^{2}\right) \nabla_{a} \nabla_{b} h^{\prime}-(2 a+b) \nabla_{a} \nabla_{b} \nabla_{c} \nabla_{d} h^{c d} \\
+\left(\frac{a_{0}}{2}+12 a H^{2}+2 b H^{2}+\frac{b}{2} \square\right)\left(\nabla_{a} \nabla^{c} h_{b c}+\nabla_{b} \nabla^{c} h_{a c}\right) \\
+\frac{1}{2} \tilde{g}_{a b}\left[\left(-a_{0}-12 a H^{2}-2 b H^{2}\right) \nabla_{c} \nabla_{d} h^{c d}+\left(a_{0}-b H^{2}\right) \square h^{\prime}\right. \\
\left.\quad+\left(a_{0}-12 a H^{2}-2 b H^{2}\right) H^{2} h^{\prime}+(4 a+b)\left(\square \nabla_{c} \nabla_{d} h^{c d}-\square^{2} h^{\prime}\right)\right]=0 .
\end{aligned}
$$

The Minkowskian correspondence of the theory can be obtained by taking the zero curvature (i.e. $H \rightarrow 0$ ) of eq. (2.14), it is

$$
\begin{aligned}
& -\frac{1}{2}\left[b \square^{2} h_{a b}+a_{0} \square h_{a b}-(4 a+b) \partial_{a} \partial_{b} \square h^{\prime}-\left(a_{0}+b \square\right)\left(\partial_{a} \partial^{c} h_{b c}+\partial_{b} \partial^{c} h_{a c}\right)+a_{0} \partial_{a} \partial_{b} h^{\prime}\right] \\
& +\frac{1}{2} \eta_{a b}\left[a_{0}\left(\square h^{\prime}-\partial_{c} \partial_{d} h^{c d}\right)+(4 a+b)\left(\square \partial_{c} \partial_{d} h^{c d}-\square^{2} h^{\prime}\right)\right]-(2 a+b) \partial_{a} \partial_{b} \partial_{c} \partial_{d} h^{c d}=0,
\end{aligned}
$$

where $\eta_{a b}$ is the metric and $\square=\eta_{a b} \partial^{a} \partial^{b}=\partial^{a} \partial_{a}$ is the wave operator in the flat space.

In order to obtain the physical sector of the model, one must to impose the physical conditions $\nabla_{a} h^{a b}=0=\nabla_{b} h^{a b}$ and $h^{\prime}=0$. In this case following Takook et al. [29] we obtain

$$
\left[-\frac{b}{2} \square^{2}-\left(\frac{a_{0}}{2}+12 a H^{2}+b H^{2}\right) \square+H^{2}\left(a_{0}+24 a H^{2}+4 b H^{2}\right)\right] h_{a b}=0,
$$

fore the metric signature $(-,+,+,+)$, and

$$
\left[-\frac{b}{2} \square^{2}+\left(\frac{a_{0}}{2}+12 a H^{2}+b H^{2}\right) \square+H^{2}\left(a_{0}+24 a H^{2}+4 b H^{2}\right)\right] h_{a b}=0,
$$

fore the metric signature $(+,-,-,-)$.

In the following subsection, in order to consider the possible relations between the field equation and the UIR's of the dS group, the linearized field equation (2.17) will be written in terms of the Casimir operators of dS group, using the 5-dimensional ambient space notations.

\section{2 dS group and Casimir operators in the field equation}

The dS space-time is a maximally symmetric space-time having a positive constant curvature. It can be easily represented as a four-dimensional hyperboloid

$$
\eta_{\alpha \beta} x^{\alpha} x^{\beta}=-H^{-2}, \quad \alpha, \beta, \ldots=0,1,2,3,4,
$$

embedded in a flat five-dimensional space with metric $\eta_{\alpha \beta}=\operatorname{diag}(1,-1,-1,-1,-1)$. The $\mathrm{dS}$ metrics is

$$
d s^{2}=\left.\eta_{\alpha \beta} d x^{\alpha} d x^{\beta}\right|_{x^{2}=-H^{-2}}=\tilde{g}_{a b} d X^{a} d X^{b}, a, b, \ldots=0,1,2,3,
$$


where $X^{a}$ 's are the 4 space-time intrinsic coordinates in dS hyperboloid. Different coordinate systems can be chosen $[30,31]$. Any geometrical object in this space can be written in terms of the four local intrinsic coordinates $X^{a}$ or in terms of the five global ambient space coordinates $x^{\alpha}$.

In order to express eq. (2.17) in terms of the ambient space notations, originally developed by Christian Fronsdal [32], we adopt the tensor field $\mathcal{K}_{\alpha \beta}(x)$ in ambient space notations. Note that the "intrinsic" field $h_{a b}(X)$ is locally determined by the transverse tensor field $\mathcal{K}_{\alpha \beta}(x)$ through

$$
h_{a b}(X)=\frac{\partial x^{\alpha}}{\partial X^{a}} \frac{\partial x^{\beta}}{\partial X^{b}} \mathcal{K}_{\alpha \beta}(x(X)) .
$$

In these notations, the solutions to the field equations are easily written out in terms of scalar fields. The reader how is not familiar to the ambient space notations is referred to [39] and references therein. The symmetric tensor field $\mathcal{K}_{\alpha \beta}(x)$ is defined on dS space-time and satisfies the transversality condition $[33,34]$

$$
x \cdot \mathcal{K}(x)=0 \text {, i.e. } x^{\alpha} \mathcal{K}_{\alpha \beta}(x)=0 \text {, and } x^{\beta} \mathcal{K}_{\alpha \beta}(x)=0 .
$$

The covariant derivative in the ambient space notations is

$$
D_{\beta} T_{\alpha_{1} \ldots \alpha_{i} \ldots \alpha_{n}}=\bar{\partial}_{\beta} T_{\alpha_{1} \ldots \alpha_{i} \ldots \alpha_{n}}-H^{2} \sum_{i=1}^{n} x_{\alpha_{i}} T_{\alpha_{1} \ldots \beta \ldots \alpha_{n}}
$$

where $\bar{\partial}$ is tangential (or transverse) derivative in dS space

$$
\bar{\partial}_{\alpha}=\theta_{\alpha \beta} \partial^{\beta}=\partial_{\alpha}+H^{2} x_{\alpha} x \cdot \partial, \quad x \cdot \bar{\partial}=0,
$$

$\theta_{\alpha \beta}=\eta_{\alpha \beta}+H^{2} x_{\alpha} x_{\beta}$ is the transverse projector. It is easily shown that the metric $\tilde{g}_{a b}$ corresponds to the transverse projector $\theta_{\alpha \beta}$ that is

$$
\tilde{g}_{a b}(X)=\frac{\partial x^{\alpha}}{\partial X^{a}} \frac{\partial x^{\beta}}{\partial X^{b}} \theta_{\alpha \beta}(x) .
$$

The kinematical group of dS space is the 10-parameter group $\mathrm{SO}_{0}(1,4)$ which is one of the two possible deformations of the Poincaré group. There are two Casimir operators

$$
Q_{s}^{(1)}=-\frac{1}{2} L_{\alpha \beta} L^{\alpha \beta}, \quad Q_{s}^{(2)}=-W_{\alpha} W^{\alpha},
$$

where

$$
W_{\alpha}=-\frac{1}{8} \epsilon_{\alpha \beta \gamma \delta \eta} L^{\beta \gamma} L^{\delta \eta}, \quad \text { with } 10 \text { infinitesimal generators } \quad L_{\alpha \beta}=M_{\alpha \beta}+S_{\alpha \beta} .
$$

The subscript $s$ in $Q_{s}^{(1)}, Q_{s}^{(2)}$ reminds that the carrier space is constituted by tensors of rank $s$. The orbital part $M_{\alpha \beta}$, and the action of the spinorial part $S_{\alpha \beta}$ on a rank-2 tensor field $\mathcal{K}$ defined on the ambient space read respectively [34]

$$
M_{\alpha \beta}=-i\left(x_{\alpha} \partial_{\beta}-x_{\beta} \partial_{\alpha}\right), \quad S_{\alpha \beta} \mathcal{K}_{\gamma \delta}=-i\left(\eta_{\alpha \gamma} \mathcal{K}_{\beta \delta}-\eta_{\beta \gamma} \mathcal{K}_{\alpha \delta}+\eta_{\alpha \delta} \mathcal{K}_{\beta \gamma}-\eta_{\beta \delta} \mathcal{K}_{\alpha \gamma}\right) .
$$


The symbol $\epsilon_{\alpha \beta \gamma \delta \eta}$ holds for the usual antisymmetrical tensor. The action of the Casimir operator $Q_{2}^{(1)}$ on $\mathcal{K}$ can be written in the more explicit form

$$
Q_{2}^{(1)} \mathcal{K}(x)=\left(Q_{0}^{(1)}-6\right) \mathcal{K}(x)+2 \eta \mathcal{K}^{\prime}+2 \mathcal{S} x \partial \cdot \mathcal{K}(x)-2 \mathcal{S} \partial x \cdot \mathcal{K}(x),
$$

where, $Q_{0}^{(1)}=-\frac{1}{2} M_{\alpha \beta} M^{\alpha \beta}=-H^{-2}(\bar{\partial})^{2}$ is the scalar Casimir operator. The symmetrizer $\mathcal{S}$ is defined for two vectors $\xi_{\alpha}$ and $\omega_{\beta}$ by $\mathcal{S}\left(\xi_{\alpha} \omega_{\beta}\right)=\xi_{\alpha} \omega_{\beta}+\xi_{\beta} \omega_{\alpha} . \mathcal{K}^{\prime}$ is the trace of the tensor $\mathcal{K}$ and the action of the Casimir operator $Q_{1}^{(1)}$ on the vector $K$ can be written in the more explicit form

$$
Q_{1}^{(1)} K(x)=\left(Q_{0}^{(1)}-2\right) K(x)+2 x \bar{\partial} \cdot K(x)+2 H^{2} x x \cdot K(x)-2 \bar{\partial} x \cdot K(x) .
$$

As shown by Dixmier [35], the UIR,s of dS group have a classification scheme in terms of a pair of parameters $(p, q)$. The Casimir operators take the following possible spectral values:

$$
\left\langle Q_{p}^{(1)}\right\rangle=-p(p+1)-(q+1)(q-2), \quad\left\langle Q_{p}^{(2)}\right\rangle=-p(p+1) q(q-1) .
$$

Depending on the different values of the pair of parameters $(p, q)$, three different series of representations are distinguishable: the principal, the complementary and the discrete series [35, 36]. Mathematical details of the group contraction and the physical principles underlying the relationship between $\mathrm{dS}$ and Poincaré groups can be found in refs. [37] and [38] respectively. The spin-2 tensor representations relevant to the present work are [39]:

i) The UIR's of the principal series labeled by $U^{2, \nu}$ with $p=s=2$ and $q=\frac{1}{2}+i \nu$ correspond to the Casimir spectral values:

$$
\left\langle Q_{2}^{(1)}\right\rangle=\nu^{2}-\frac{15}{4}, \nu \in \mathbb{R}
$$

note that $U^{2, \nu}$ and $U^{2,-\nu}$ are equivalent.

ii) The UIR's of the complementary series denoted by $V^{2, q}$ with $p=s=2$ and $q-q^{2}=\mu$, correspond to the following spectral values

$$
\left\langle Q_{2}^{(1)}\right\rangle=q-q^{2}-4 \equiv \mu-4, \quad 0<\mu<\frac{1}{4} .
$$

iii) The UIR's of the discrete series conventionally labeled by $\Pi_{2, q}^{ \pm}$in which $p=s=2$ and takes the following spectral values

$$
\left\langle Q_{2}^{(1)}\right\rangle=-6-(q+1)(q-2), \quad q=1,2 .
$$

The "massless" spin-2 field in dS space corresponds to the $\Pi_{2,2}^{ \pm}$and $\Pi_{2,1}^{ \pm}$cases in which the $\operatorname{sign} \pm$, stands for the helicity. In these cases, the two representations $\Pi_{2,2}^{ \pm}$, in the discrete series with $p=q=2$, have a Minkowskian interpretation. It is important to note that the representations $\Pi_{2,1}^{ \pm}$do not have corresponding flat limit [39]. (More details can be found in [34] and references therein.) 
We now attempt to express the wave equation (2.17) in terms of the Casimir operators of dS group. The d'Alembertian operator becomes [40]

$$
\square h_{a b}=\nabla^{c} \nabla_{c} h_{a b}=\frac{\partial x^{\alpha}}{\partial X^{a}} \frac{\partial x^{\beta}}{\partial X^{b}}\left[-H^{2} Q_{0}^{(1)}-2 H^{2}\right] \mathcal{K}_{\alpha \beta},
$$

and

$$
\square^{2} h_{a b}=\nabla^{c} \nabla_{c} \nabla^{d} \nabla_{d} h_{a b}=\frac{\partial x^{\alpha}}{\partial X^{a}} \frac{\partial x^{\beta}}{\partial X^{b}}\left[H^{4}\left(Q_{0}^{(1)}\right)^{2}+4 H^{4} Q_{0}^{(1)}+4 H^{4}\right] \mathcal{K}_{\alpha \beta},
$$

where, the conditions of tracelessness and divergence free (e.i. $\bar{\partial} . \mathcal{K}=0=\mathcal{K}^{\prime}$ ), have been imposed to the physical states. By use of the above equations in eq. (2.17) we have

$$
\left[\frac{b}{2} H^{2}\left(Q_{0}^{(1)}\right)^{2}+\left(\frac{a_{0}}{2}+12 a H^{2}+3 b H^{2}\right) Q_{0}^{(1)}\right] \mathcal{K}_{\alpha \beta}=0 .
$$

In terms of different choice of coefficients in the proposed action (2.1) different gravitational theories may be achieved. Now the following various choices are considerable

- By choosing $a=0$ and $b=0$ we return to the physical linear pure dS theory, that is

$$
Q_{0}^{(1)} \mathcal{K}_{\alpha \beta}=0, \quad \text { or } \quad\left(Q_{2}^{(1)}+6\right) \mathcal{K}_{\alpha \beta}=0 .
$$

This is an eigen-value equation with the eigen-value $\left\langle Q_{2}^{(1)}\right\rangle=-6$. From the group theoretical point of view this corresponds to UIR's of dS group labeled by $\Pi_{2,2}^{ \pm}$in the discrete series which reduces to the physical representations of the Poincaré group in the zero curvature limit. This is why it is called as the physical state. It has been discussed in [39], for the gauge-fixed value equal to zero, [41] for the gauge-fixed value equal to $\frac{2}{5}$ and the extended discussions are given in [42].

- Letting $a_{0}=1, b=0$, the model reduces to a $f(\mathcal{R})$ theory model with $f(\mathcal{R})=$ $\mathcal{R}+a \mathcal{R}^{2}$. It is known as a relatively successful model, which explains the inflation and positive acceleration of the universe [43-46]. Under these conditions, the linearized field equation (2.36) reduces to

$$
\left(1+24 a H^{2}\right) Q_{0}^{(1)} \mathcal{K}_{\alpha \beta}=0, \quad \text { or } \quad\left(1+24 a H^{2}\right)\left(Q_{2}^{(1)}+6\right) \mathcal{K}_{\alpha \beta}=0 .
$$

It corresponds to the UIR's of dS group labeled by $\Pi_{2,2}^{ \pm}$in the discrete series too. This is why the model is a successful one. The field equation (2.38) has been considered in ref. [47].

- One may set $a_{0}=0, a=-\frac{1}{3}$ and $b=1$, by which the theory reduces to the Weyl conformal theory with the linearized field equation

$$
Q_{0}^{(1)}\left(Q_{0}^{(1)}-2\right) \mathcal{K}_{\alpha \beta}=0, \quad \text { or } \quad\left(Q_{2}^{(1)}+6\right)\left(Q_{2}^{(1)}+4\right) \mathcal{K}_{\alpha \beta}=0 .
$$

The same equation has been obtained by Dehghani, et al. from a different approach in [39]. 
The field equation $\left(Q_{2}^{(1)}+4\right) \mathcal{K}_{\alpha \beta}=0$, is also an eigen-value equation with the eigenvalue $\left\langle Q_{2}^{(1)}\right\rangle=-4$. It corresponds to one of the UIR's of dS group denoted by $\Pi_{2,1}^{ \pm}$in the discrete series with the same Poincare correspondence as $\Pi_{2,2}^{ \pm}$in the zero curvature limit. Indeed two of UIR's of dS group have only one Poincaré correspondence. It has been discussed in [48].

As it is clear with the help of above-mentioned examples, we believe that it is necessary for any successful theory of gravity to transform according to the UIR,s of dS group. In other words if a model of modified gravity theory does not correspond to the UIR,s of dS group in its linear approximations it can not produce valid and helpful physical results.

For the general discussion on the proposed modified gravity theory, let $A=a H^{2}$ and $B=b H^{2}$. In terms of these dimensionless coefficients the field equation (2.36) can be written as

$$
\left[\left(Q_{0}^{(1)}\right)^{2}+\left(\frac{a_{0}}{B}+24 \frac{A}{B}+6\right) Q_{0}^{(1)}\right] \mathcal{K}_{\alpha \beta}=0, \quad B \neq 0 .
$$

As a direct mathematical result, the proposed model in it's linear approximation, generally transforms according to the UIR's of dS group and it is a suitable candidate model of gravitation on $\mathrm{dS}$ space if the characteristic equation

$$
\frac{a_{0}}{B}+24 \frac{A}{B}+8=0, \quad B \neq 0,
$$

is satisfied. Under this condition it describes a massless spin-2 particle (the graviton, if it exists) in it's linear approximation and transforms according to two of UIR,s of dS group. We therefore believe that it can be a successful modified gravity theory. For more clarity, in the following sections, we solve the field equation (2.40), with the condition (2.41), using the ambient space formalism. Also we obtain the two-point function for the linearized theory of gravitation making use of the ambient space notations, and show that the results are free of any theoretical problems.

\section{Solution to the conformal field equation}

A general solution of to the conformall field equation can be constructed from the combination of a scalar field and two vector fields. Let us first introduce a traceless and transverse tensor field $\mathcal{K}$ in terms of a five-dimensional constant vector $Z_{1}=\left(Z_{1 \alpha}\right)$ and a scalar field $\phi_{1}$ and two vector fields $K$ and $K_{g}$ by putting [34, 39, 41, 42, 48, 49]

$$
\mathcal{K}=\theta \phi_{1}+\mathcal{S} \bar{Z}_{1} K+D_{2} K_{g},
$$

where $D_{2}$ is the generalized gradient operator defined by $D_{2} K=\mathcal{S}\left(D_{1}+x\right) K, D_{1 \alpha}=$ $H^{-2} \bar{\partial}_{\alpha}$ and $\bar{Z}_{1 \alpha}=\theta_{\alpha \beta} Z_{1}^{\beta}$. Taking the trace of $\mathcal{K}_{\alpha \beta}$ we have

$$
\mathcal{K}^{\prime}=4 \phi_{1}+2 Z_{1} . K+2 H^{2}\left(x . Z_{1}\right) x \cdot K+2 D_{1} \cdot K_{g}-2 x \cdot K_{g}=0,
$$

Using the ansatz (3.1) to the field equation we have (appendix F)

$$
\left\{\begin{array}{l}
\left(Q_{0}^{(1)}+4\right)\left(Q_{0}^{(1)}+6\right) \phi_{1}+8\left(Q_{0}^{(1)}+2\right) Z_{1} \cdot K=0, \\
Q_{1}^{(1)}\left(Q_{1}^{(1)}+2\right) K=0, \quad \text { or } \quad Q_{1}^{(1)} Q_{0}^{(1)} K=0, \quad \partial \cdot K=0=x \cdot K \\
\left(Q_{1}^{(1)}+4\right)\left(Q_{1}^{(1)}+6\right) K_{g}=4 H^{2}\left[\left(Q_{1}^{(1)}+5\right) x \cdot Z_{1} K+Z_{1} \cdot D_{1} K-x Z_{1} \cdot K\right] .
\end{array}\right.
$$


The vector field $K$ can be written in the following general form

$$
K_{\alpha}=\bar{Z}_{2 \alpha} \phi_{2}+D_{1 \alpha} \phi_{3},
$$

where $Z_{2}$ is another constant 5-vector and $\phi_{2}$ and $\phi_{3}$ are two arbitrary scalar fields, should be determined. Using the divergenceless condition we have

$$
Q_{0}^{(1)} \phi_{3}=Z_{2} . \bar{\partial} \phi_{2}+4 H^{2}\left(x \cdot Z_{2}\right) \phi_{2},
$$

and substituting eq. (3.4) in eq. (3.3)(b) leads to the following two equations

$$
\begin{aligned}
& Q_{0}^{(1)}\left(Q_{0}^{(1)}-2\right) \phi_{2}=0, \\
& Q_{0}^{(1)}\left(Q_{0}^{(1)}+2\right) \phi_{3}=4 H^{2} Q_{0}^{(1)}\left[\left(x . Z_{2}\right) \phi_{2}\right]+8 H^{2}\left(x . Z_{2}\right) \phi_{2}+4 Z_{2} . \bar{\partial} \phi_{2} .
\end{aligned}
$$

The eq. (3.6) has a dS plane wave solution of the form

$$
\phi_{2}=(H x . \xi)^{\sigma}, \quad \xi^{2}=0, \quad \text { with } \quad \sigma(\sigma+3)(\sigma+2)(\sigma+1)=0 .
$$

Note that $\phi_{2}$ is the minimally coupled scalar field for $\sigma=0,-3$. In that case it obeys the field equation $Q_{0}^{(1)} \phi_{2}=0[39,50]$. Also $\phi_{2}$ is the conformally coupled scalar field for $\sigma=-1,-2$ and satisfies the field equation $\left(Q_{0}^{(1)}-2\right) \phi_{2}=0[51]$.

Substituting $Q_{0}^{(1)} \phi_{3}$ and $\left(Q_{0}^{(1)}\right)^{2} \phi_{3}$ from eq. (3.5) into eq. (3.7), we obtain

$$
Q_{0}^{(1)} Z_{2} . \bar{\partial} \phi_{2}=2 Z_{2} . \bar{\partial} \phi_{2}
$$

Now regarding eqs. (3.6) and (3.9) and using the identity

$$
Q_{0}^{(1)}\left[\left(x . Z_{2}\right) \phi_{2}\right]=\left(x . Z_{2}\right) Q_{0}^{(1)} \phi_{2}-4\left(x . Z_{2}\right) \phi_{2}-2 Z_{2} . D_{1} \phi_{2},
$$

we obtain

$$
Q_{0}^{(1)}\left[\left(x . Z_{2}\right) Q_{0}^{(1)} \phi_{2}\right]=2 Q_{0}^{(1)}\left[\left(x . Z_{2}\right) \phi_{2}\right]+8\left(x . Z_{2}\right) \phi_{2}+4 Z_{2} \cdot D_{1} \phi_{2} .
$$

Combining eqs. (3.9) and (3.11) we have

$$
\left(x . Z_{2}\right) \phi_{2}=\frac{1}{8} Q_{0}^{(1)}\left[\left(x . Z_{2}\right) Q_{0}^{(1)} \phi_{2}-2\left(x . Z_{2}\right) \phi_{2}-2 Z_{2} . D_{1} \phi_{2}\right] .
$$

Substituting eqs. (3.9) and (3.12) in eq. (3.5) we have

$$
\phi_{3}=\frac{1}{2}\left[H^{2}\left(x . Z_{2}\right) Q_{0}^{(1)} \phi_{2}-Z_{2} . \bar{\partial} \phi_{2}-2 H^{2}\left(x . Z_{2}\right) \phi_{2}\right] .
$$

Now the solution to eq. (3.3)(b) can be written in terms of the dS massless scalar field $\phi_{2} \equiv \phi_{s}$ as

$$
K_{\alpha}=\bar{Z}_{2 \alpha} \phi_{s}+\frac{1}{2} D_{1 \alpha}\left[H^{2}\left(x . Z_{2}\right) Q_{0}^{(1)}-Z_{2} . \bar{\partial}-2 H^{2} x . Z_{2}\right] \phi_{s} .
$$

The explicit form of the vector field $K_{\alpha}$ is

$$
K=\frac{\sigma}{2}\left[(\sigma+2) \bar{Z}_{2}+\left(\sigma^{2}+2 \sigma-2\right) \frac{x \cdot Z_{2}}{x \cdot \xi} \bar{\xi}\right] \phi_{s},
$$


and the condition of $\bar{\partial} . K_{\alpha}=0$ can be written in the following explicit form

$$
\bar{\partial} . K=\frac{1}{2} \sigma^{2}(\sigma+3)(\sigma+4)\left(x . Z_{1}\right)\left(x . Z_{2}\right) \phi_{s}=0 .
$$

Noting eq. (3.8), it is valid only for $\sigma=0,-3$. As pointed out before we can treat the scalar field $\phi_{s}$ as the massless minimally coupled scalar field. Furthermore under these circumstances the vector field $K_{\alpha}$ satisfies the relation

$$
Q_{0}^{(1)} K_{\alpha}=0, \quad \text { or } \quad\left(Q_{1}^{(1)}+2\right) K=0 .
$$

It is easy to show that eq. (3.3)(a) has a solution of the form

$$
\phi_{1}=-\frac{2}{3} Z_{1} \cdot K, \quad Q_{0}^{(1)}\left(Q_{0}^{(1)}-2\right) \phi_{1}=0 .
$$

It means that $\phi_{1}$ satisfies the massless minimally coupled scalar field equation in dS space [50, 51]. Now eq. (3.2) can be written as

$$
\bar{\partial} . K_{g}=\frac{1}{3} H^{2} Z_{1} \cdot K, \quad x . K_{g}=0 .
$$

Making use of the relation

$$
\left(Q_{1}^{(1)}+5\right) x \cdot Z_{1} K=2 x\left(Z_{1} \cdot K\right)-2\left(Z_{1} \cdot D_{1}\right) K-\left(x \cdot Z_{1}\right) K,
$$

eq. $(3.3)(c)$ can be written as

$$
\left(Q_{1}^{(1)}+4\right)\left(Q_{1}^{(1)}+6\right) K_{g}=4 H^{2}\left[x Z_{1} \cdot K-x \cdot Z_{1} K-Z_{1} \cdot D_{1} K\right] .
$$

Now using the identities

$$
\begin{aligned}
6\left(x \cdot Z_{1}\right) K & =\left(Q_{1}^{(1)}+6\right)\left[\left(x \cdot Z_{1}\right) K+\frac{1}{9} D_{1}\left(Z_{1} \cdot K\right)\right], \\
2\left(x Z_{1} \cdot K-Z_{1} \cdot D_{1} K\right) & =\left(Q_{1}^{(1)}+6\right)\left(x \cdot Z_{1} K\right),
\end{aligned}
$$

in eq. (3.20) we obtain

$$
\left(Q_{1}^{(1)}+4\right) K_{g}=\frac{4}{3} H^{2}\left[\left(x \cdot Z_{1}\right) K-\frac{1}{18} D_{1}\left(Z_{1} \cdot K\right)\right] .
$$

It is easy to show that

$$
\begin{aligned}
4 D_{1}\left(Z_{1} \cdot K\right) & =\left(Q_{1}^{(1)}+4\right) D_{1}\left(Z_{1} \cdot K\right) \\
4\left(x \cdot Z_{1}\right) K & =\left(Q_{1}^{(1)}+4\right)\left[\left(x \cdot Z_{1}\right) K+\frac{1}{6} D_{1}\left(Z_{1} \cdot K\right)\right] .
\end{aligned}
$$

Combining eqs. (3.21)-(3.23) results in

$$
K_{g}=\frac{1}{3} H^{2}\left[\left(x \cdot Z_{1}\right) K+\frac{1}{9} D_{1}\left(Z_{1} \cdot K\right)\right] .
$$

It satisfies the conditions given in eq. (3.19). 
Substituting eqs. (3.15), (3.18) and (3.24) in eq. (3.1) one can show that

$$
\mathcal{K}_{\alpha \beta}(x)=\mathcal{E}_{\alpha \beta}\left(x, \xi, Z_{1}, Z_{2}\right) \phi_{s},
$$

where $\phi_{s}$ is a massless scalar field in $\mathrm{dS}$ space and $\mathcal{E}$ is a generalized symmetric polarization tensor,

$$
\mathcal{E}=\frac{\sigma}{2}\left[-\frac{2}{3} \theta Z_{1} \cdot+\mathcal{S} \bar{Z}_{1}+H^{2} \frac{1}{3} D_{2}\left(x \cdot Z_{1}+\frac{1}{9} D_{1} Z_{1} \cdot\right)\right]\left[(\sigma+2) \bar{Z}_{2}+\left(\sigma^{2}+2 \sigma-2\right) \frac{x \cdot Z_{2}}{x \cdot \xi} \bar{\xi}\right] .
$$

It is consistent with the results in [39] and [42] with $c=0$.

\section{The conformal two-point function}

The two-point function $\mathcal{W}_{\alpha \beta \alpha^{\prime} \beta^{\prime}}\left(x, x^{\prime}\right)$, which is a solution of the wave equation with respect to $x$ or $x^{\prime}$, can be found simply in terms of the scalar two-point function. Very similar to the recurrence formula (3.1) let us try the following possibility [34, 39, 41, 42, 48, 49]

$$
\mathcal{W}\left(x, x^{\prime}\right)=\theta \theta^{\prime} \mathcal{W}_{0}\left(x, x^{\prime}\right)+\mathcal{S S}^{\prime} \theta \cdot \theta^{\prime} \mathcal{W}_{1}\left(x, x^{\prime}\right)+D_{2} D_{2}^{\prime} \mathcal{W}_{g}\left(x, x^{\prime}\right)
$$

where $\mathcal{W}, \mathcal{W}_{1}$ and $\mathcal{W}_{g}$ are transverse bi-vectors, $\mathcal{W}_{0}$ is bi-scalar and $D_{2} D_{2}^{\prime}=D_{2}^{\prime} D_{2}$. Substituting the two-point function (4.1) in the field equation with respect to $x$, we have

$$
\left\{\begin{array}{l}
\left(Q_{0}^{(1)}+4\right)\left(Q_{0}^{(1)}+6\right) \theta^{\prime} \mathcal{W}_{0}+8\left(Q_{0}^{(1)}+2\right) \mathcal{S}^{\prime} \theta^{\prime} . \mathcal{W}_{1} \\
Q_{1}^{(1)}\left(Q_{1}^{(1)}+2\right) \mathcal{W}_{1}=0, \quad \text { or } \quad Q_{1}^{(1)} Q_{0}^{(1)} \mathcal{W}_{1}=0, \quad \partial . \mathcal{W}_{1}=0 \\
\left(Q_{1}^{(1)}+4\right)\left(Q_{1}^{(1)}+6\right) D_{2}^{\prime} \mathcal{W}_{g}=4 H^{2} \mathcal{S}^{\prime}\left[\left(Q_{1}^{(1)}+5\right)\left(x . \theta^{\prime}\right) \mathcal{W}_{1}+\theta^{\prime} . D_{1} \mathcal{W}_{1}+x \theta^{\prime} . \mathcal{W}_{1}\right]
\end{array}\right.
$$

The solution to eq. (4.2)(b) has the following general form

$$
\mathcal{W}_{1}=\theta \cdot \theta^{\prime} \mathcal{W}_{2}+D_{1} D_{1}^{\prime} \mathcal{W}_{3}, \quad \text { and } \quad D_{1}^{\prime} \mathcal{W}_{3}=\frac{1}{2}\left[H^{2}\left(x \cdot \theta^{\prime}\right) Q_{0}^{(1)}-\theta^{\prime} . \bar{\partial}-2 H^{2} x \cdot \theta^{\prime}\right] \mathcal{W}_{2}
$$

in which $\mathcal{W}_{2} \equiv \mathcal{W}_{s}$ is the massless minimally coupled scalar two-point function. The dS-invariance two-point function for the massless minimally coupled scalar field in the "Gupta-Bleuler vacuum" state is [52]

$$
\mathcal{W}_{s}\left(x, x^{\prime}\right)=\frac{i H^{2}}{8 \pi^{2}} \epsilon\left(x^{0}-x^{\prime 0}\right)\left[\delta\left(1-\mathcal{Z}\left(x, x^{\prime}\right)\right)+\vartheta\left(\mathcal{Z}\left(x, x^{\prime}\right)-1\right)\right]
$$

with

$$
\mathcal{Z}=-H^{2} x \cdot x^{\prime}, \quad \text { and } \quad \epsilon\left(x^{0}-x^{\prime 0}\right)=\left\{\begin{aligned}
1 & x^{0}>x^{0} \\
0 & x^{0}=x^{\prime 0} \\
-1 & x^{0}<x^{\prime 0}
\end{aligned}\right.
$$

In summary, the solution to the above system of equations is

$$
\begin{aligned}
\mathcal{W}_{1} & =\left[\theta \cdot \theta^{\prime}+\frac{1}{2} D_{1}\left(H^{2} x \cdot \theta^{\prime} Q_{0}^{(1)}-\theta^{\prime} . \bar{\partial}-2 H^{2} x \cdot \theta^{\prime}\right)\right] \mathcal{W}_{s}, \\
\theta^{\prime} \mathcal{W}_{0}\left(x, x^{\prime}\right) & =-\frac{2}{3} \mathcal{S}^{\prime} \theta^{\prime} \cdot \mathcal{W}_{1}\left(x, x^{\prime}\right), \\
D_{2}^{\prime} \mathcal{W}_{g}\left(x, x^{\prime}\right) & =\frac{1}{3} H^{2} \mathcal{S}^{\prime}\left[\left(x \cdot \theta^{\prime}\right) \mathcal{W}_{1}+\frac{1}{9} D_{1}\left(\theta^{\prime} \cdot \mathcal{W}_{1}\right)\right] .
\end{aligned}
$$


The two-point function (4.1) also satisfies the field equation with respect to $x^{\prime}$, in this case one can obtain

$$
\left\{\begin{array}{l}
\left(Q_{0}^{\prime(1)}+4\right)\left(Q_{0}^{\prime(1)}+6\right) \theta \mathcal{W}_{0}+8\left(Q_{0}^{\prime(1)}+2\right) \mathcal{S} \theta \cdot \mathcal{W}_{1}, \\
Q_{1}^{\prime(1)}\left(Q_{1}^{\prime(1)}+2\right) \mathcal{W}_{1}=0, \quad \text { or } \quad Q_{1}^{\prime(1)} Q_{0}^{\prime(1)} \mathcal{W}_{1}=0, \quad \partial^{\prime} . \mathcal{W}_{1}=0 \\
\left(Q_{1}^{\prime(1)}+4\right)\left(Q_{1}^{\prime(1)}+6\right) D_{2} \mathcal{W}_{g}=4 H^{2} \mathcal{S}\left[\left(Q_{1}^{\prime(1)}+5\right)\left(x^{\prime} . \theta\right) \mathcal{W}_{1}+\theta \cdot D_{1}^{\prime} \mathcal{W}_{1}-x^{\prime} \theta \cdot \mathcal{W}_{1}\right] \cdot(c)
\end{array}\right.
$$

with the solutions

$$
\begin{aligned}
\mathcal{W}_{1} & =\left[\theta^{\prime} \cdot \theta+\frac{1}{2} D_{1}^{\prime}\left(H^{2} x^{\prime} \cdot \theta Q_{0}^{\prime(1)}-\theta \cdot \bar{\partial}^{\prime}-2 H^{2} x^{\prime} \cdot \theta\right)\right] \mathcal{W}_{s}, \\
\theta \mathcal{W}_{0}\left(x, x^{\prime}\right) & =-\frac{2}{3} \mathcal{S} \theta \cdot \mathcal{W}_{1}\left(x, x^{\prime}\right) \\
D_{2} \mathcal{W}_{g}\left(x, x^{\prime}\right) & =\frac{1}{3} H^{2} \mathcal{S}\left[\left(x^{\prime} . \theta\right) \mathcal{W}_{1}+\frac{1}{9} D_{1}^{\prime}\left(\theta \cdot \mathcal{W}_{1}\right)\right] .
\end{aligned}
$$

Note that the primed operators act on the primed coordinates only.

Making use of eqs. (4.6)-(4.8) or (4.10)-(4.12) one can show that the conformal twopoint function can be written as

$$
\mathcal{W}_{\alpha \beta \alpha^{\prime} \beta^{\prime}}=\Delta_{\alpha \beta \alpha^{\prime} \beta^{\prime}} \mathcal{W}_{s}
$$

where

$$
\begin{aligned}
\Delta=\frac{1}{6}\left[-2 \theta \mathcal{S}^{\prime} \theta^{\prime} .+\mathcal{S S}^{\prime} \theta \cdot \theta^{\prime}+H^{2} D_{2} \mathcal{S}^{\prime}\left(x \cdot \theta^{\prime}+\frac{1}{3} D_{1} \theta^{\prime} .\right)\right] \\
\times\left[2 \theta \cdot \theta^{\prime}+D_{1}\left(H^{2} x \cdot \theta^{\prime} Q_{0}^{(1)}-\theta^{\prime} . \bar{\partial}-2 H^{2} x \cdot \theta^{\prime}\right)\right] .
\end{aligned}
$$

It agrees with the results in [39] and [42] with $c=0$.

\section{Conclusion}

According to the recent cosmological observations it seems that the standard Einstein theory of gravity may be incomplete and many attempts have been made to modify this theory. The so-called modified theory of gravitation and, in particular, non-linear gravity theories or higher-order theories of gravity have provided interesting results. The proposed models are based on gravitational actions which are non-linear in the Ricci curvature and constructed out by curvature invariants.

This work is devoted to an extension of the Einstein-Hilbert gravitational action, which is constructed out by the linear combination of Ricci scalar and Ricci tensor invariants in dS space. Varying the proposed action with respect to metric tensor leads to a fourth order gravitational field equation, conventionally named as the modified gravitational theory. The background field method is utilized and the linearized field equation is obtained in terms of intrinsic coordinates in the 4-dimensional dS space as the background. 
The gravitational field in the linear approximation behaves like a massless spin-2 particle which propagates on the background space-time. According to Wigner's theorem, a linear gravitational field should transform according to the UIR's of the symmetry group of the background space-time. In order to investigate the possible relations between the field equation and the UIR's of dS group it is transformed into the flat five-dimensional ambient space and the linearized field equation is written in terms of the Casimir operators of $\mathrm{dS}$ group. We obtained the Minkowskian correspondence of the theory by taking the zero curvature limit. The physical sector of the theory is obtained by imposing the divergenceless and traceless conditions. Some interesting theories are reproduced as the special cases of the theory and their validity and successfulness are discussed from group theoretical point of view. We demonstrated that it is necessary for a theory to be successful, in dS space-time, if it transforms according to the UIR's of dS group. We showed that the proposed theory transforms according to the UIR,s of dS group if the constant coefficients satisfy some simple conditions. As a result this theory can be used as a successful model for solving the problems in the framework of quantum gravity.

As an special case of the theory the linearized Weyl theory of gravity is reproduced which transforms according to two of the UIR's of dS group denoted by $\Pi_{2,2}^{ \pm}$and $\Pi_{2,1}^{ \pm}$ in discrete series. We obtained the solution to the conformally invariant field equation, using the ambient space notations. The solution can be written as the multiplication of a symmetric rank-2 generalized polarization tensor and a massless minimally coupled scalar field in dS space. Also we have calculated the conformally invariant two-point function, in terms of the basic bi-vectors of the ambient space. It is dS invariant, symmetric and satisfies the traceless and divergenceless conditions. We therefore claim that the proposed modified gravity theory under the given restrictions is a successful one and the introduced procedure can be used as a theoretical testing for the validity and successfulness of any given modified theory of gravity.

\section{A Some useful mathematical relations}

The following relations have been used in deriving the linearized field equations.

$$
\begin{aligned}
\tilde{R}_{a b c d} & =H^{2}\left(\tilde{g}_{a c} \tilde{g}_{b d}-\tilde{g}_{a d} \tilde{g}_{b c}\right), \\
\tilde{R}_{a b} & =3 H^{2} \tilde{g}_{a b}, \\
\tilde{R} & =12 H^{2}, \\
\left(\mathcal{R}_{d a b}^{c}\right)_{L} & \equiv \delta R_{d a b}^{c}=\frac{1}{2}\left[\nabla_{a}\left(\nabla_{d} h_{b}^{c}+\nabla_{b} h_{d}^{c}-\nabla^{c} h_{d b}\right)\right. \\
\left.-\nabla_{b}\left(\nabla_{d} h_{a}^{c}+\nabla_{a} h_{d}^{c}-\nabla^{c} h_{a d}\right)\right] . & \left.-2 H^{2} h^{\prime} \tilde{g}_{a b}-\square h_{a b}-\nabla_{a} \nabla_{b} h^{\prime}\right) . \\
\left(\mathcal{R}_{a b}\right)_{L} & \equiv \delta R_{a b}=\frac{1}{2}\left(\nabla_{a} \nabla_{c} h_{b}^{c}+\nabla_{b} \nabla_{c} h_{a}^{c}+8 H^{2} h_{a b}\right. \\
(\mathcal{R})_{L} & \equiv \delta R=\nabla_{c} \nabla_{b} h^{c b}-\square h^{\prime}-3 H^{2} h^{\prime} .
\end{aligned}
$$




$$
\begin{gathered}
\left(\mathcal{R}_{d}^{c}\right)_{L} \equiv \delta R_{d}^{c}=\frac{1}{2}\left(\nabla^{c} \nabla_{a} h_{d}^{a}+\nabla_{d} \nabla_{a} h^{a c}+8 H^{2} h_{d}^{c}\right. \\
\left.-2 H^{2} h^{\prime} \tilde{g}_{d}^{c}-\square h_{d}^{c}-\nabla^{c} \nabla_{d} h^{\prime}\right)-3 H^{2} h_{d}^{c} . \\
\left(\mathcal{R}^{b c}\right)_{L} \equiv \delta R^{b c}=\frac{1}{2}\left(\nabla^{c} \nabla_{a} h^{a b}+\nabla^{b} \nabla_{a} h^{a c}+8 H^{2} h^{b c}\right. \\
\left.-2 H^{2} h^{\prime} \tilde{g}^{b c}-\square h^{b c}-\nabla^{c} \nabla^{b} h^{\prime}\right)-6 H^{2} h^{b c} . \\
\left(\nabla_{a} \nabla_{b} \mathcal{R}\right)_{L} \equiv \delta \nabla_{a} \nabla_{b} R=\nabla_{a} \nabla_{b} \delta R=\nabla_{a} \nabla_{b}\left(\nabla_{c} \nabla_{d} h^{c d}-\square h^{\prime}-3 H^{2} h^{\prime}\right) . \\
(\square \mathcal{R})_{L} \equiv \delta \square R=\square \delta R=\square\left(\nabla_{c} \nabla_{d} h^{c d}-\square h^{\prime}-3 H^{2} h^{\prime}\right) . \\
\left(\nabla_{a} \nabla_{b} \mathcal{R}_{c d}\right)_{L} \equiv \delta \nabla_{a} \nabla_{b} R_{c d}=\frac{1}{2} \nabla_{a} \nabla_{b}\left(\nabla_{c} \nabla_{e} h_{d}^{e}+\nabla_{d} \nabla_{e} h_{c}^{e}\right. \\
\left.+2 H^{2} h_{c d}-2 H^{2} h^{\prime} \tilde{g}_{c d}-\square h_{c d}-\nabla_{c} \nabla_{d} h^{\prime}\right) . \\
\left(\nabla_{a} \nabla_{b} \mathcal{R}_{d}^{c}\right)_{L} \equiv \delta \nabla_{a} \nabla_{b} R_{d}^{c}=\frac{1}{2} \nabla_{a} \nabla_{b}\left(\nabla^{c} \nabla_{e} h_{d}^{e}+\nabla_{d} \nabla_{e} h^{c e}\right. \\
\left.+2 H^{2} h_{d}^{c}-2 H^{2} h^{\prime} \tilde{g}_{d}^{c}-\square h_{d}^{c}-\nabla^{c} \nabla_{d} h^{\prime}\right) \\
\left(\nabla_{a} \nabla_{b} \mathcal{R}^{c d}\right)_{L} \equiv \delta \nabla_{a} \nabla_{b} R^{c d}=\frac{1}{2} \nabla_{a} \nabla_{b}\left(\nabla^{c} \nabla_{e} h^{e d}+\nabla^{d} \nabla_{e} h^{c e}\right. \\
\left.+2 H^{2} h^{c d}-2 H^{2} h^{\prime} \tilde{g}^{c d}-\square h^{c d}-\nabla^{c} \nabla^{d} h^{\prime}\right) . \\
\left(\square \mathcal{R}_{c d}\right)_{L} \equiv \delta \square R_{c d}=\frac{1}{2} \square\left(\nabla_{c} \nabla_{e} h_{d}^{e}+\nabla_{d} \nabla_{e} h_{c}^{e}\right. \\
\left.+2 H^{2} h_{c d}-2 H^{2} h^{\prime} \tilde{g}_{c d}-\square h_{c d}-\nabla_{c} \nabla_{d} h^{\prime}\right) .
\end{gathered}
$$

\section{B Details of derivation of eq. (2.2)}

In this subsection we obtain the field equation through variation of the action. The action (2.1) can be written as

$$
\begin{aligned}
I & =\frac{1}{16 \pi G} \int d^{4} x \sqrt{-g}\left[f(\mathcal{R})+b \mathcal{R}^{a b} \mathcal{R}_{a b}\right], \quad f(\mathcal{R})=a_{0}(\mathcal{R}-2 \Lambda)+a \mathcal{R}^{2}, \\
\delta[\sqrt{-g} f(\mathcal{R})] & =f(\mathcal{R}) \delta \sqrt{-g}+\sqrt{-g} f^{\prime}(\mathcal{R}) \delta \mathcal{R} \\
\delta \sqrt{-g} & =-\frac{1}{2} \sqrt{-g} g_{a b} \delta g^{a b} \\
\delta \mathcal{R} & =\delta\left(g^{a b} \mathcal{R}_{a b}\right)=\mathcal{R}_{a b} \delta g^{a b}+g^{a b} \delta \mathcal{R}_{a b}, \\
\delta \mathcal{R}_{a b} & =\nabla_{c} \delta \Gamma_{a b}^{c}-\nabla_{b} \delta \Gamma_{a c}^{c} .
\end{aligned}
$$

The statement in eq. (B.5) is the difference of two connections, it transforms as a tensor. one can show that

$$
\delta \Gamma_{a b}^{c}=\frac{1}{2} g^{c d}\left(\nabla_{a} \delta g_{b d}+\nabla_{b} \delta g_{a d}-\nabla_{d} \delta g_{a b}\right),
$$

and substituting in eq. (B.5)we have

$$
\delta \mathcal{R}_{a b}=\frac{1}{2}\left(\nabla^{c} \nabla_{a} \delta g_{b c}+\nabla^{c} \nabla_{b} \delta g_{a c}-\nabla_{a} \nabla_{b} g^{c d} \delta g_{c d}-\square \delta g_{a b}\right)
$$


Now return to eq. (B.4) we have

$$
\delta \mathcal{R}=\mathcal{R}_{a b} \delta g^{a b}+g_{a b} \square \delta g^{a b}-\nabla_{a} \nabla_{b} \delta g^{a b} .
$$

Using eq. (B.3) and eq. (B.8) in eq. (B.2) we have

$$
\begin{aligned}
\delta[\sqrt{-g} f(\mathcal{R})] & =\sqrt{-g}\left[f^{\prime}(\mathcal{R}) \mathcal{R}_{a b}-\frac{1}{2} g_{a b} f(\mathcal{R})+f^{\prime}(\mathcal{R})\left(g_{a b} \square-\nabla_{a} \nabla_{b}\right)\right] \delta g^{a b} . \\
\delta\left(\mathcal{R}^{a b} \mathcal{R}_{a b}\right) & =\delta\left(g^{c a} g^{b d} \mathcal{R}_{c d} \mathcal{R}_{a b}\right)=2\left(\mathcal{R}_{c}^{a} \mathcal{R}_{a b} \delta g^{b c}+\mathcal{R}^{a b} \delta \mathcal{R}_{a b}\right)
\end{aligned}
$$

and noting eq. (B.7) we can show

$$
\delta\left(\mathcal{R}^{a b} \mathcal{R}_{a b}\right)=2 \mathcal{R}_{c}^{a} \mathcal{R}_{a b} \delta g^{b c}+\mathcal{R}^{a b}\left(\nabla^{c} \nabla_{a} \delta g_{b c}+\nabla^{c} \nabla_{b} \delta g_{a c}-\nabla_{a} \nabla_{b} g^{c d} \delta g_{c d}-\square \delta g_{a b}\right) .
$$

Now it is easy to show that

$$
\begin{aligned}
\delta\left(\sqrt{-g} \mathcal{R}^{a b} \mathcal{R}_{a b}\right)=\sqrt{-g}\left[-\frac{1}{2} g_{a b} \mathcal{R}_{c d} \mathcal{R}^{c d}+2 \mathcal{R}_{a}^{c} \mathcal{R}_{c b}-\mathcal{R}_{a}^{c} \nabla_{b} \nabla_{c}\right. \\
\left.-\mathcal{R}_{b}^{c} \nabla_{a} \nabla_{c}+\mathcal{R}^{c d} \nabla_{c} \nabla_{d} g_{a b}+\mathcal{R}_{a b} \square\right] \delta g^{a b} . \\
\delta I=\frac{1}{16 \pi G} \int d^{4} x \sqrt{-g}\left[f^{\prime}(\mathcal{R}) \mathcal{R}_{a b}-\frac{1}{2} g_{a b} f(\mathcal{R})-\frac{b}{2} g_{a b} \mathcal{R}_{c d} \mathcal{R}^{c d}\right. \\
+2 b \mathcal{R}_{a}^{c} \mathcal{R}_{c b}+f^{\prime}(\mathcal{R}) g_{a b} \square-f^{\prime}(\mathcal{R}) \nabla_{a} \nabla_{b}-b \mathcal{R}_{a}^{c} \nabla_{b} \nabla_{c} \\
\left.-b \mathcal{R}_{b}^{c} \nabla_{a} \nabla_{c}+b \mathcal{R}^{c d} \nabla_{c} \nabla_{d} g_{a b}+b \mathcal{R}_{a b} \square\right] \delta g^{a b} .
\end{aligned}
$$

Doing integration by part on the last six terms two times leads to

$$
\begin{aligned}
\delta I=\frac{1}{16 \pi G} \int & d^{4} x \sqrt{-g}\left[f^{\prime}(\mathcal{R}) \mathcal{R}_{a b}-\frac{1}{2} g_{a b} f(\mathcal{R})-\frac{b}{2} g_{a b} \mathcal{R}_{c d} \mathcal{R}^{c d}\right. \\
& +2 b \mathcal{R}_{a}^{c} \mathcal{R}_{c b}+g_{a b} \square f^{\prime}(\mathcal{R})-\nabla_{a} \nabla_{b} f^{\prime}(\mathcal{R})-b \nabla_{c} \nabla_{b} \mathcal{R}_{a}^{c} \\
& \left.-b \nabla_{c} \nabla_{a} \mathcal{R}_{b}^{c}+b \nabla_{c} \nabla_{d} \mathcal{R}^{c d} g_{a b}+b \square \mathcal{R}_{a b}\right] \delta g^{a b} .
\end{aligned}
$$

Noting that action remains invariant under variation of the metric and putting $\delta I=0$, results in eq. (2.2).

\section{Details of derivation of eq. (2.7)}

Now $\mathcal{H}_{a b}^{(0)}$ can be written as

$$
\begin{aligned}
\mathcal{H}_{a b}^{(0)} & =\tilde{R}_{a b}+\delta R_{a b}-\frac{1}{2}(\tilde{R}+\delta R)\left(\tilde{g}_{a b}+h_{a b}\right)+\Lambda\left(\tilde{g}_{a b}+h_{a b}\right) \\
& =\tilde{H}_{a b}^{(0)}+\delta R_{a b}-\frac{1}{2}\left(\tilde{R} h_{a b}+\tilde{g}_{a b} \delta R\right)+\Lambda h_{a b}+\text { nonlinear terms. }
\end{aligned}
$$

Using eqs. (A.3), (A.5) and (A.6) in (C.1) leads to the expression presented in eq. (2.7). 


\section{Details of derivation of eq. (2.9)}

Now, $\mathcal{H}_{a b}^{(1)}$ can be written as

$$
\begin{aligned}
\mathcal{H}_{a b}^{(1)}+2(\tilde{R}+\delta R) & \left(\tilde{R}_{a b}+\delta R_{a b}\right)-2\left(\nabla_{a} \nabla_{b} \tilde{R}+\delta \nabla_{a} \nabla_{b} R\right) \\
& -\frac{1}{2}\left(\tilde{g}_{a b}+h_{a b}\right)\left[\left(\tilde{R}^{2}+2 \tilde{R} \delta R\right)-4(\square \tilde{R}+\delta \square R)\right],
\end{aligned}
$$

which may be written as

$$
\begin{aligned}
\mathcal{H}_{a b}^{(1)}= & \tilde{H}_{a b}^{(1)}-2\left(\delta \nabla_{a} \nabla_{b} R-\tilde{R} \delta R_{a b}-\tilde{R}_{a b} \delta R\right) \\
& -\tilde{g}_{a b}(\tilde{R} \delta R-2 \delta \square R)-\frac{1}{2} h_{a b} \tilde{R}^{2}+\text { nonlinear terms. }
\end{aligned}
$$

Making use of eqs. (A.5), (A.6), (A.9) and (A.10) we have $\mathcal{H}_{a b}^{(1)}=\tilde{H}_{a b}^{(1)}+H_{a b}^{(1)}$, and $H_{a b}^{(1)}$ is the same as given in eq. (2.9).

\section{E Details of derivation of eq. (2.11)}

Eq. (2.4) can be written in the following form

$$
\begin{aligned}
\mathcal{H}_{a b}^{(2)}= & \left(\square \tilde{R}_{a b}+\delta \square R_{a b}\right)-\left(\nabla_{c} \nabla_{a} \tilde{R}_{b}^{c}+\delta \nabla_{c} \nabla_{a} R_{b}^{c}\right)-\left(\nabla_{c} \nabla_{b} \tilde{R}_{a}^{c}+\delta \nabla_{c} \nabla_{b} R_{a}^{c}\right) \\
& -\frac{1}{2}\left(\tilde{g}_{a b}+h_{a b}\right)\left[\left(\tilde{R}^{c d}+\delta R^{c d}\right)\left(\tilde{R}_{c d}+\delta R_{c d}\right)-2\left(\nabla_{c} \nabla_{d} \tilde{R}^{c d}+\delta \nabla_{c} \nabla_{d} R^{c d}\right)\right] \\
& +2\left(\tilde{R}_{a}^{c}+\delta R_{a}^{c}\right)\left(\tilde{R}_{c b}+\delta R_{c b}\right) .
\end{aligned}
$$

It can be written as

$$
\begin{aligned}
\mathcal{H}_{a b}^{(2)}= & \tilde{H}_{a b}^{(2)}+\delta \square R_{a b}-\delta \nabla_{c} \nabla_{a} R_{b}^{c}-\delta \nabla_{c} \nabla_{b} R_{a}^{c}+2\left(\tilde{R}_{a}^{c} \delta R_{c b}+\tilde{R}_{c b} \delta R_{a}^{c}\right) \\
& -\frac{1}{2} \tilde{g}_{a b}\left(\tilde{R}^{c d} \delta R_{c d}+\tilde{R}_{c d} \delta R^{c d}-2 \delta \nabla_{c} \nabla_{d} R^{c d}\right) \\
& -\frac{1}{2} h_{a b} \tilde{R}^{c d} \tilde{R}_{c d}+\text { nonlinear terms. }
\end{aligned}
$$

Now using the relations given in appendix A we can show that

$$
\begin{aligned}
\tilde{R}_{a}^{c} \delta R_{c b}= & \frac{3}{2} H^{2}\left(\nabla_{a} \nabla_{c} h_{b}^{c}+\nabla_{b} \nabla_{c} h_{a}^{c}+8 H^{2} h_{a b}-2 H^{2} h^{\prime} \tilde{g}_{a b}-\square h_{a b}-\nabla_{a} \nabla_{b} h^{\prime}\right), \\
\tilde{R}_{c b} \delta R_{a}^{c}= & \frac{3}{2} H^{2}\left(\nabla_{a} \nabla_{c} h_{b}^{c}+\nabla_{b} \nabla_{c} h_{a}^{c}+2 H^{2} h_{a b}-2 H^{2} h^{\prime} \tilde{g}_{a b}-\square h_{a b}-\nabla_{a} \nabla_{b} h^{\prime}\right), \\
\delta \nabla_{c} \nabla_{d} R^{c d}= & \nabla_{c} \nabla_{d}\left(\nabla^{c} \nabla_{f} h^{f d}+\nabla^{d} \nabla_{f} h^{f c}\right)+2 H^{2} \nabla_{c} \nabla_{d} h^{c d} \\
& -2 H^{2} \square h^{\prime}-\nabla_{c} \nabla_{d} \square h^{c d}-\nabla_{c} \square \nabla^{c} h^{\prime}, \\
\tilde{R}^{c d} \delta R_{c d}= & 3 H^{2}\left(\nabla_{c} \nabla_{d} h^{c d}-\square h^{\prime}\right), \\
\tilde{R}_{c d} \delta R^{c d}= & 3 H^{2}\left(\nabla_{c} \nabla_{d} h^{c d}-\square h^{\prime}-6 H^{2} h^{\prime}\right) .
\end{aligned}
$$

Substituting in eq. (E.2) we obtain $\mathcal{H}_{a b}^{(2)}=\tilde{H}_{a b}^{(2)}+H_{a b}^{(2)}$ and $H_{a b}^{(2)}$ is the statement given in eq. (2.11). 
In derivation steps, the following identities have been used

$$
\begin{aligned}
\nabla_{c} \nabla_{a} \square h_{b}^{c}= & \square \nabla_{a} \nabla_{c} h_{b}^{c}+2 H^{2} \nabla_{a} \nabla_{c} h_{b}^{c}-2 H^{2} \nabla_{b} \nabla_{c} h_{a}^{c}+2 H^{2} \tilde{g}_{a b} \nabla_{c} \nabla_{d} h^{c d} \\
& -2 H^{2} \nabla_{a} \nabla_{b} h^{\prime}+4 H^{2} \square h_{a b}-H^{2} \tilde{g}_{a b} \square h^{\prime}, \\
\nabla_{c} \nabla_{a} \nabla^{c} \nabla_{d} h_{b}^{d}= & \square \nabla_{a} \nabla_{d} h_{b}^{d}-H^{2} \nabla_{b} \nabla_{d} h_{c}^{d}+H^{2} \tilde{g}_{a b} \nabla_{c} \nabla_{d} h^{c d}, \\
\nabla_{c} \nabla_{a} \nabla_{b} \nabla^{c} h^{\prime}= & \square \nabla_{a} \nabla_{b} h^{\prime}-H^{2} \nabla_{a} \nabla_{b} h^{\prime}+H^{2} \tilde{g}_{a b} \square h^{\prime}, \\
\nabla_{c} \nabla_{a} \nabla_{b} \nabla_{d} h^{c d}= & \nabla_{a} \nabla_{b} \nabla_{c} \nabla_{d} h^{c d}+4 H^{2} \nabla_{a} \nabla_{c} h_{b}^{c}+3 H^{2} \nabla_{b} \nabla_{c} h_{a}^{c}-H^{2} \tilde{g}_{a b} \nabla_{c} \nabla_{d} h^{c d}, \\
\square \nabla_{a} \nabla_{b} h^{\prime}= & \nabla_{a} \nabla_{b} \square h^{\prime}+8 H^{2} \nabla_{a} \nabla_{b} h^{\prime}-2 H^{2} \tilde{g}_{a b} \square h^{\prime} .
\end{aligned}
$$

\section{F Details of derivation of eq. (3.3)}

By imposing the tensor field

$$
\mathcal{K}=\theta \phi_{1}+\mathcal{S} \bar{Z}_{1} K+D_{2} K_{g}
$$

to obey the field equation

$$
\left(Q_{2}^{(1)}+4\right)\left(Q_{2}^{(1)}+6\right) \mathcal{K}=0,
$$

and making use of the following identities [34],

$$
\begin{aligned}
Q_{2}^{(1)}(\theta \phi) & =\theta Q_{0}^{(1)} \phi, \\
Q_{2}^{(1)} D_{2} K_{g} & =D_{2} Q_{1}^{(1)} K_{g}, \\
Q_{2}^{(1)} \mathcal{S}(\bar{Z} K) & =\mathcal{S}\left[\bar{Z}\left(Q_{1}^{(1)}-4\right) K\right]-2 H^{2} D_{2}(x . Z) K+4 \theta Z . K,
\end{aligned}
$$

we have

$$
\begin{aligned}
\left(Q_{2}^{(1)}+4\right)([ & \left.\left(Q_{0}^{(1)}+6\right) \phi_{1}+4 Z_{1} \cdot K\right] \theta \\
& \left.+\mathcal{S}\left[\bar{Z}_{1}\left(Q_{1}^{(1)}+2\right) K\right]+D_{2}\left[\left(Q_{1}^{(1)}+6\right) K_{g}-2 H^{2}\left(x \cdot Z_{1}\right) K\right]\right)=0 .
\end{aligned}
$$

Making use of eqs. (F.3)-(F.5) in eq. (F.6) once again, we obtain

$$
\begin{array}{r}
\theta\left[\left(Q_{0}^{(1)}+4\right)\left(Q_{0}^{(1)}+6\right) \phi_{1}+4 Q_{0}^{(1)} Z_{1} \cdot K+4\left(Q_{1}^{(1)}+2\right) x \cdot Z_{1} K+16 Z_{1} \cdot K\right] \\
+\mathcal{S} \bar{Z}_{1}\left[Q_{1}^{(1)}\left(Q_{1}^{(1)}+2\right) K\right]+D_{2}\left[\left(Q_{1}^{(1)}+4\right)\left(Q_{1}^{(1)}+6\right) K_{g}\right. \\
\left.-2 H^{2}\left(x \cdot Z_{1}\right)\left(Q_{1}^{(1)}+6\right) K-2 H^{2} Q_{1}^{(1)}\left(x \cdot Z_{1} K\right)\right]=0 .
\end{array}
$$

Using the conditions $x . K=0=\partial . K$ in eq. (2.29) we have

$$
\left(Q_{1}^{(1)}+2\right) K=Q_{0}^{(1)} K,
$$

from which we can write

$$
Q_{0}^{(1)}\left(Z_{1} \cdot K\right)=\left(Q_{1}^{(1)}+2\right) Z_{1} \cdot K
$$


Substituting (F.9) in eq. (F.7) results in

$$
\begin{aligned}
& \theta\left[\left(Q_{0}^{(1)}+4\right)\left(Q_{0}^{(1)}+6\right) \phi_{1}+8 Q_{0}^{(1)} Z_{1} \cdot K+16 Z_{1} \cdot K\right]+\mathcal{S} \bar{Z}_{1}\left[Q_{1}^{(1)}\left(Q_{1}^{(1)}+2\right) K\right] \\
& \quad+D_{2}\left[\left(Q_{1}^{(1)}+4\right)\left(Q_{1}^{(1)}+6\right) K_{g}-2 H^{2}\left(x \cdot Z_{1}\right)\left(Q_{1}^{(1)}+6\right) K-2 H^{2} Q_{1}^{(1)}\left(x \cdot Z_{1} K\right)\right]=0 .
\end{aligned}
$$

It is easy to show that

$$
Q_{1}^{(1)}\left(x . Z_{1} K\right)=x \cdot Z_{1}\left(Q_{1}^{(1)}-4\right) K-2 Z_{1} \cdot D_{1} K+2 x Z_{1} \cdot K .
$$

Now combining eqs. (F.10) and (F.11) leads to the following equation

$$
\begin{aligned}
\theta[ & \left.\left(Q_{0}^{(1)}+4\right)\left(Q_{0}^{(1)}+6\right) \phi_{1}+8\left(Q_{0}^{(1)}+2\right) Z_{1} \cdot K\right]+\mathcal{S} \bar{Z}_{1}\left[Q_{1}^{(1)}\left(Q_{1}^{(1)}+2\right) K\right] \\
& +D_{2}\left[\left(Q_{1}^{(1)}+4\right)\left(Q_{1}^{(1)}+6\right) K_{g}-4 H^{2}\left(\left(Q_{1}^{(1)}+5\right)\left(x \cdot Z_{1} K\right)+Z_{1} \cdot D_{1} K-x Z_{1} \cdot K\right)\right]=0
\end{aligned}
$$

which results in eq. (3.3).

Open Access. This article is distributed under the terms of the Creative Commons Attribution License (CC-BY 4.0), which permits any use, distribution and reproduction in any medium, provided the original author(s) and source are credited.

\section{References}

[1] WMAP collaboration, D.N. Spergel et al., First year Wilkinson Microwave Anisotropy Probe (WMAP) observations: determination of cosmological parameters, Astrophys. J. Suppl. 148 (2003) 175 [astro-ph/0302209] [INSPIRE].

[2] WMAP collaboration, D.N. Spergel et al., Wilkinson Microwave Anisotropy Probe (WMAP) three year results: implications for cosmology, Astrophys. J. Suppl. 170 (2007) 377 [astro-ph/0603449] [INSPIRE].

[3] SDSS collaboration, M. Tegmark et al., Cosmological parameters from SDSS and WMAP, Phys. Rev. D 69 (2004) 103501 [astro-ph/0310723] [InSPIRE].

[4] SDSS collaboration, U. Seljak et al., Cosmological parameter analysis including SDSS Ly- $\alpha$ forest and galaxy bias: constraints on the primordial spectrum of fluctuations, neutrino mass and dark energy, Phys. Rev. D 71 (2005) 103515 [astro-ph/0407372] [InSPIRE].

[5] SDSS collaboration, D.J. Eisenstein et al., Detection of the baryon acoustic peak in the large-scale correlation function of SDSS luminous red galaxies, Astrophys. J. 633 (2005) 560 [astro-ph/0501171] [INSPIRE].

[6] B. Jain and A. Taylor, Cross-correlation tomography: measuring dark energy evolution with weak lensing, Phys. Rev. Lett. 91 (2003) 141302 [astro-ph/0306046] [INSPIRE].

[7] S. Capozziello, V.F. Cardone, S. Carloni and A. Troisi, Curvature quintessence matched with observational data, Int. J. Mod. Phys. D 12 (2003) 1969 [astro-ph/0307018] [INSPIRE].

[8] A.A. Starobinsky, A new type of isotropic cosmological models without singularity, Phys. Lett. B 91 (1980) 99 [INSPIRE]. 
[9] S. Capozziello, V.F. Cardone and A. Troisi, Dark energy and dark matter as curvature effects, JCAP 08 (2006) 001 [astro-ph/0602349] [INSPIRE].

[10] K.-i. Maeda and N. Ohta, Inflation from M-theory with fourth-order corrections and large extra dimensions, Phys. Lett. B 597 (2004) 400 [hep-th/0405205] [INSPIRE].

[11] K.-i. Maeda and N. Ohta, Inflation from superstring/M theory compactification with higher order corrections. 1., Phys. Rev. D 71 (2005) 063520 [hep-th/0411093] [InSPIRE].

[12] N. Ohta, Accelerating cosmologies and inflation from M/superstring theories, Int. J. Mod. Phys. A 20 (2005) 1 [hep-th/0411230] [InSPIRE].

[13] K. Akune, K.-i. Maeda and N. Ohta, Inflation from superstring/M-theory compactification with higher order corrections. II. Case of quartic Weyl terms, Phys. Rev. D 73 (2006) 103506 [hep-th/0602242] [INSPIRE].

[14] S. Nojiri and S.D. Odintsov, Modified $f(R)$ gravity consistent with realistic cosmology: from matter dominated epoch to dark energy universe, Phys. Rev. D 74 (2006) 086005 [hep-th/0608008] [INSPIRE].

[15] S. Nojiri and S.D. Odintsov, Modified Gauss-Bonnet theory as gravitational alternative for dark energy, Phys. Lett. B 631 (2005) 1 [hep-th/0508049] [INSPIRE].

[16] S. Capozziello, S. Nojiri and S.D. Odintsov, Unified phantom cosmology: Inflation, dark energy and dark matter under the same standard, Phys. Lett. B 632 (2006) 597 [hep-th/0507182] [INSPIRE].

[17] S. Nojiri and S.D. Odintsov, Where new gravitational physics comes from: M-theory?, Phys. Lett. B 576 (2003) 5 [hep-th/0307071] [INSPIRE].

[18] S. Nojiri and S.D. Odintsov, Modified gravity with negative and positive powers of curvature: Unification of inflation and cosmic acceleration, Phys. Rev. D 68 (2003) 123512 [InSPIRE].

[19] S. Nojiri, S.D. Odintsov and M. Sasaki, Gauss-Bonnet dark energy, Phys. Rev. D 71 (2005) 123509 [hep-th/0504052] [INSPIRE].

[20] R. Kerner, Cosmology without singularity and nonlinear gravitational Lagrangians, Gen. Rel. Grav. 14 (1982) 453 [INSPIRE].

[21] J.P. Duruisseau and R. Kerner, The effective gravitational lagrangian and the energy momentum tensor in the inflationary universe, Class. Quant. Grav. 3 (1986) 817 [INSPIRE].

[22] P. Teyssandier, Linearized $R+R^{2}$ gravity: a new gauge and new solutions, Class. Quant. Grav. 6 (1989) 219 [inSPIRE].

[23] G. Magnano, M. Ferraris and M. Francaviglia, Nonlinear gravitational Lagrangians, Gen. Rel. Grav. 19 (1987) 465 [INSPIRE].

[24] Supernova Search Team collaboration, A.G. Riess et al., Observational evidence from supernovae for an accelerating universe and a cosmological constant, Astron. J. 116 (1998) 1009 [astro-ph/9805201] [INSPIRE].

[25] Supernova Cosmology Project collaboration, S. Perlmutter et al., Measurements of $\Omega$ and $\Lambda$ from 42 high redshift supernovae, Astrophys. J. 517 (1999) 565 [astro-ph/9812133] [INSPIRE].

[26] Boomerang collaboration, P. de Bernardis et al., A flat universe from high resolution maps of the cosmic microwave background radiation, Nature 404 (2000) 955 [astro-ph/0004404] [INSPIRE]. 
[27] WMAP collaboration, C.L. Bennett et al., First year Wilkinson Microwave Anisotropy Probe (WMAP) observations: preliminary maps and basic results, Astrophys. J. Suppl. 148 (2003) 1 [astro-ph/0302207] [INSPIRE].

[28] Z.-Y. Fan and H. Lü, Thermodynamical first laws of black holes in quadratically-extended gravities, Phys. Rev. D 91 (2015) 064009 [arXiv:1501.00006] [INSPIRE].

[29] M.V. Takook, M.R. Tanhayi and S. Fatemi, Conformal linear gravity in de Sitter space, J. Math. Phys. 51 (2010) 032503 [arXiv:0903.5249] [INSPIRE].

[30] E. Mottola, Particle creation in de Sitter space, Phys. Rev. D 31 (1985) 754 [INSPIRE].

[31] B. Allen and A. Folacci, The massless minimally coupled scalar field in de Sitter space, Phys. Rev. D 35 (1987) 3771 [INSPIRE].

[32] C. Fronsdal, Singletons and massless, integral spin fields on de Sitter space (Elementary particles in a curved space. 7), Phys. Rev. D 20 (1979) 848 [InSPIRE].

[33] P.A.M. Dirac, The electron wave equation in de-Sitter space, Annals Math. 36 (1935) 657 [INSPIRE].

[34] T. Garidi, J.P. Gazeau and M.V. Takook, 'Massive' spin two field in de Sitter space, J. Math. Phys. 44 (2003) 3838 [hep-th/0302022] [INSPIRE].

[35] J. Dixmier, Représentations intégrables du groupe de de Sitter, Bull. Soc. Math. France 89 (1961) 9 .

[36] B. Takahashi, Sur les represéntations unitaires des groupes de Lorentz généralisés, Bull. Soc. Math. France 91 (1963) 289.

[37] J. Levy-Nahas, Deformation and contraction of Lie algebra, J. Math. Phys. 8 (1967) 1211.

[38] H. Bacry and J. Levy-Leblond, Possible kinematics, J. Math. Phys. 9 (1968) 1605 [INSPIRE].

[39] M. Dehghani, S. Rouhani, M.V. Takook and M.R. Tanhayi, Conformally invariant 'massless' spin-2 field in the de Sitter universe, Phys. Rev. D 77 (2008) 064028 [arXiv:0805.2227] [INSPIRE].

[40] M.V. Takook and M.R. Tanhayi, Linear Weyl gravity in de Sitter universe, JHEP 12 (2010) 044 [arXiv: 0903.2670] [INSPIRE].

[41] M. Dehghani, Covariant two-point function for massless spin-2 field in de Sitter space, Mod. Phys. Lett. A 24 (2009) 3283 [inSPIRE].

[42] M. Dehghani, Evaluation of the graviton two-point function in de Sitter space, Int. J. Mod. Phys. A 25 (2010) 3749 [INSPIRE].

[43] B. Whitt, Fourth order gravity as general relativity plus matter, Phys. Lett. B 145 (1984) 176 [INSPIRE].

[44] V. Muller, H.J. Schmidt and A.A. Starobinsky, The stability of the de Sitter space-time in fourth order gravity, Phys. Lett. B 202 (1988) 198 [inSPIRE].

[45] J.D. Barrow and S. Cotsakis, Inflation and the conformal structure of higher order gravity theories, Phys. Lett. B 214 (1988) 515 [INSPIRE].

[46] K.-i. Maeda, Towards the Einstein-Hilbert action via conformal transformation, Phys. Rev. D 39 (1989) 3159 [INSPIRE].

[47] M. Dehghani, Linear higher order gravity in de Sitter background, J. Theor. Appl. Phys. 5 (2011) 29. 
[48] M. Dehghani, A new physical state for de Sitter linear gravity, Int. J. Mod. Phys. A 26 (2010) 301 [INSPIRE].

[49] M.V. Takook, H. Pejham and M.R. Tanhahi, Conformal linear gravity in de Sitter space II, Eur. Phys. J. C 72 (2012) 2052 [arXiv:1105.3060] [INSPIRE].

[50] J.P. Gazeau, J. Renaud and M.V. Takook, Gupta-Bleuler quantization for minimally coupled scalar fields in de Sitter space, Class. Quant. Grav. 17 (2000) 1415 [gr-qc/9904023] [INSPIRE].

[51] S. Behroozi, S. Rouhani, M.V. Takook and M.R. Tanhayi, Conformally invariant wave equations and massless fields in de Sitter spacetime, Phys. Rev. D 74 (2006) 124014 [gr-qc/0512105] [INSPIRE].

[52] M.V. Takook, Covariant two point function for minimally coupled scalar field in de Sitter space-time, Mod. Phys. Lett. A 16 (2001) 1691 [gr-qc/0005020] [InSPIRE]. 